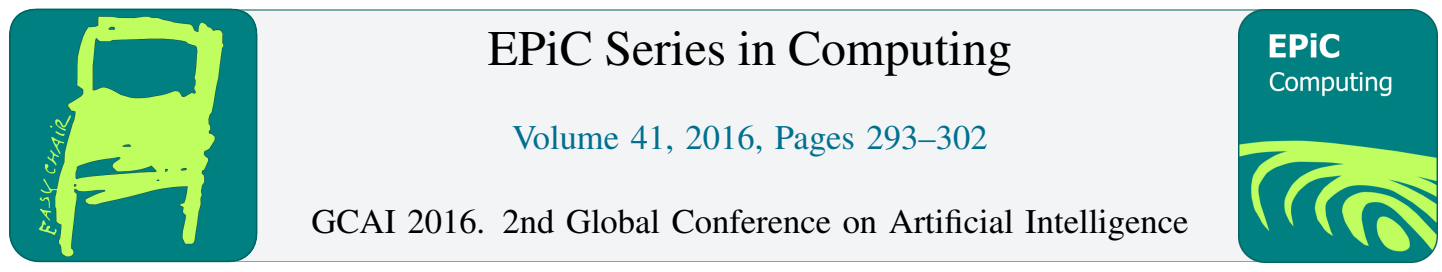

\title{
Deep Incremental Boosting
}

\author{
Alan Mosca ${ }^{1}$ and George D Magoulas ${ }^{1}$ \\ Department of Computer Science and Information Systems, Birkbeck, University of London. \{a.mosca, \\ gmagoulas\}@dcs.bbk.ac.uk
}

\begin{abstract}
This paper introduces Deep Incremental Boosting, a new technique derived from AdaBoost, specifically adapted to work with Deep Learning methods, that reduces the required training time and improves generalisation. We draw inspiration from Transfer of Learning approaches to reduce the start-up time to training each incremental Ensemble member. We show a set of experiments that outlines some preliminary results on some common Deep Learning datasets and discuss the potential improvements Deep Incremental Boosting brings to traditional Ensemble methods in Deep Learning.
\end{abstract}

\section{Introduction}

AdaBoost [9] is considered a successful Ensemble method and is commonly used in combination with traditional Machine Learning algorithms, especially Boosted Decision Trees [3]. One of the main principles behind it is the additional emphasis given to the so-called hard to classify examples from a training set.

Deep Neural Networks have also had great success on many visual problems, and there are a number of benchmark datasets in this area where the state-of-the-art results are held by some Deep Learning algorithm [12, 4].

Ideas from Transfer of Learning have found applications in Deep Learning; for example, in Convolutional Neural Networks (CNNs), when sub-features learned early in the training process can be carried forward to a new $\mathrm{CNN}$ in order to improve generalisation on a new problem of the same domain [13]. It has also been shown that these Transfer of Learning methods reduce the "warm-up" phase of the training, where a randomly-initialised CNN would have to re-learn basic feature selectors from scratch.

In this paper, we explore the synergy of AdaBoost and Transfer of Learning to accelerate this initial warm-up phase of training each new round of boosting. The proposed method, named Deep Incremental Boosting, exploits additional capacity embedded into each new round of boosting, which increases the generalisation without adding much training time. When tested in Deep Learning benchmarks, the new method is able to beat traditional Boosted CNNs on benchmark datasets, in a shorter training time.

The paper is structured as follows. Section 2 presents an overview of prior work on which the new development is based. Section 3 presents the new learning algorithm. Section 4 reports the methodology of our preliminary experimentation and the results. Section 5 provides examples where state-of-the-art models have been used as the base classifiers for Deep Incremental Boosting. Lastly, Section 6 makes conclusions on our experiments, and shows possible avenues for further development. 


\section{Prior Work}

This section gives an overview of previous work and algorithms on which our new method is based.

\subsection{AdaBoost}

AdaBoost [9] is a well-known Ensemble method, which has a proven track record of improving performance. It is based on the principle of training Ensemble members in "rounds", and at each round increasing the importance of training examples that were misclassified in the previous round. The final Ensemble is then aggregated using weights $\alpha_{0 . . N}$ calculated during the training. Algorithm 1 shows the common AdaBoost.M2 [10] variant. This variant is generally considered better for multi-class problems, such as those used in our experimentation, however the same changes we apply to AdaBoost.M2 can be applied to any other variant of AdaBoost.

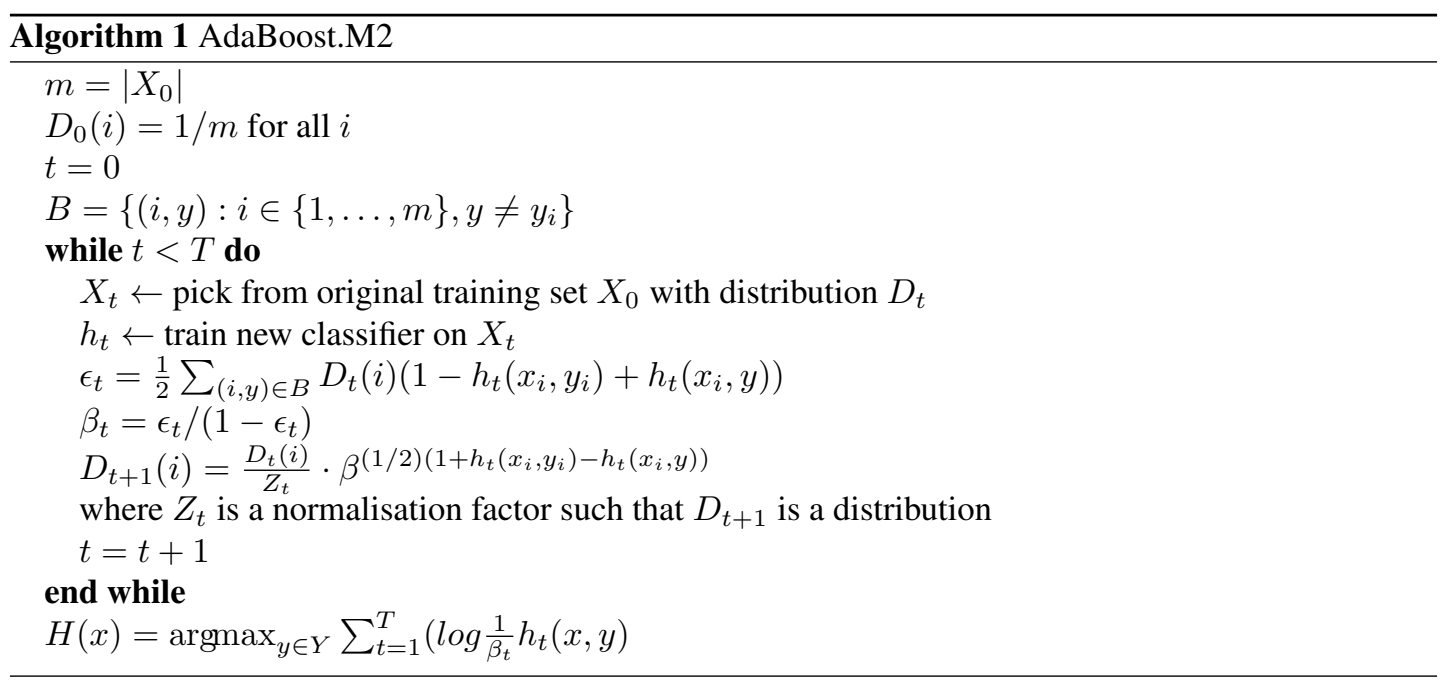

\subsection{Transfer of Learning applied to Deep Neural Networks}

Over the last few years a lot of progress has been made in the Deep Networks area due to their ability to represent features at various levels of resolution. A recent study analysed how the low-layer features of Deep Networks are transferable and can be considered general in the problem domain of image recognition [13]. More specifically it has been found that, for example, the first-layer of a CNN tends to learn filters that are either similar to Gabor filters or color blobs. Ref [2] studies Transfer of Learning in an unsupervised setting on Deep Neural Networks and also reached a similar conclusion.

In supervised Deep Learning contexts, transfer of learning can be achieved by setting the initial weights of some of the layers of a Deep Neural Network to those of a previously-trained network. Because of the findings on the generality of the first few layers of filters, this is traditionally applied mostly to those first few layers. The training is then continued on the new dataset, with the benefit that the already-learned initial features provide a much better starting position than randomly initialised weights, and as such the generalisation power is improved and the time required to train the network is reduced. 


\section{Deep Incremental Boosting}

\subsection{Motivation}

Traditional AdaBoost methods, and related variants, re-train a new classifier from scratch every round. While this, combined with the weighted re-sampling of the training set, appears at first glance to be one of the elements that create diversity in the final Ensemble, it may not be necessary to re-initialize the Network from scratch at every round.

It has already been previously shown that weights can be transferred between Networks, and in particular between subsets of a network, to accelerate the initial training phase. In the case of Convolutional Neural Networks, this particular approach is particularly fruitful, as the lower layers (those closest to the input) tend to consistently develop similar features.

\subsection{Applying Transfer of Learning to AdaBoost}

Intuition 1. Because each subsequent round of AdaBoost increases the importance given to the errors made at the previous round, the network $h_{t}$ at a given round can be repurposed at round $t+1$ to learn the newly resampled training set.

In order for this to make sense, it is necessary to formulate a few conjectures.

Definition 1. Let $X_{a}$ be a set composed of $n$ training example vectors $X_{a}=\left\{\boldsymbol{x}_{a, 1}, \boldsymbol{x}_{a, 2}, \ldots \boldsymbol{x}_{a, n}\right\}$, with its corresponding set of correct label vectors $Y_{a}=\left\{\boldsymbol{y}_{a, 1}, \boldsymbol{y}_{a, 2}, \ldots \boldsymbol{y}_{a, n}\right\}$

Definition 2. A training set $X_{a}$ is mostly similar to another set $X_{b}$ if the sets of unique instances $X_{a}$ and $X_{b}$ have more common than different elements, and the difference set is smaller than an arbitrary significant amount $\epsilon$.

This can be expressed equivalently as:

$$
\begin{array}{r}
\left|X_{a} \cap X_{b}\right|>>\left|X_{a} \ominus X_{b}\right| \\
\left|X_{a} \cap X_{b}\right|>>\left|X_{a}-X_{b}\right|+\left|X_{b}-X_{a}\right|
\end{array}
$$

or

$$
\left|X_{a} \cup X_{b}\right|=\left|X_{a} \cap X_{b}\right|+\epsilon
$$

Given the Jaccard Distance

$$
J(A, B)=\frac{|A \cap B|}{|A \cup B|}
$$

this can be formulated as

$$
J\left(X_{a}, X_{b}\right) \geq 1-\epsilon
$$

Conjecture 1. At a round of Boosting $t+1$, the resampled training set $X_{t+1}$ and the previous resampled training set $X_{t}$ are mostly similar, as in Definition 2:

$$
\left|X_{t} \cap X_{t+1}\right|>>\left|X_{t}-X_{t+1}\right|+\left|X_{t+1}-X_{t}\right|
$$

or

$$
\left|X_{t} \cup X_{t+1}\right|=\left|X_{t} \cap X_{t+1}\right|+\epsilon
$$


If we relax $\epsilon$ to be as large as we like, In the case of Boosting, we know this to be true because both $X_{t}$ and $X_{t+1}$ are resampled from $X_{0}$ with the weighting $D_{t+1}$ from the initial dataset $X_{t=0}$, so the unique sets $X_{t}$ and $X_{t+1}$ are large resampled subsets of the initial training set $X_{t=0}$ :

$$
\begin{array}{r}
X_{t} \subseteq X_{t=0} \\
X_{t+1} \subseteq X_{t=0} \\
\left|X_{t}\right|=\left|X_{t+1}\right|=\left|X_{t=0}\right|
\end{array}
$$

Definition 3. We introduce a mistake function $E\left(h_{a}, X_{b}\right)$ which counts the number of mistakes by the classifier $h_{a}$ on dataset $X_{b}$ :

$$
E\left(h_{a}, X_{b}, Y\right)=\left|\left\{\boldsymbol{x}_{b, i} \mid h_{a}\left(\boldsymbol{x}_{b, i}\right) \neq \boldsymbol{y}_{i} \forall \boldsymbol{x}_{b, i} \in X_{b}\right\}\right|
$$

where $y_{i}$ is the ground truth for example $i$, taken from the correct label set $y$.

Conjecture 2. Given Conjecture 1 and provided that the dataset $X_{t}$ and $X_{t+1}$ are mostly similar as per Definition 2, a classifier $h_{t}$ that classifies $X_{t}$ better than randomly will still perform better than randomly on a new dataset $X_{t+1}$.

Given that all sets are of the same size by definition, as they are resampled that way, we can ignore the fact that the error count on a dataset $E\left(h_{t}, X_{t}\right)$ would need to be divided by the size of the dataset $\left|X_{t}\right|$, thus simplifying the notation.

We can therefore redefine the errors made by $h_{t}$ on both $X_{t}$ and $X_{t+1}$ as:

$$
\begin{aligned}
E\left(h_{t}, X_{t}, Y\right) & =E\left(h_{t}, X_{t} \cap X_{t+1}, Y\right)+E\left(h_{t}, X_{t}-X_{t+1}, Y\right) \\
E\left(h_{t}, X_{t+1}, Y\right) & =E\left(h_{t}, X_{t} \cap X_{t+1}, Y\right)+E\left(h_{t}, X_{t+1}-X_{t}, Y\right)
\end{aligned}
$$

From Conjecture 1, the last two terms are negligible, leaving:

$$
\begin{array}{r}
E\left(h_{t}, X_{t}, Y\right)=E\left(h_{t}, X_{t} \cap X_{t+1}, Y\right)+\epsilon_{t} \\
E\left(h_{t}, X_{t+1}, Y\right)=E\left(h_{t}, X_{t} \cap X_{t+1}, Y\right)+\epsilon_{t+1}
\end{array}
$$

therefore $E\left(h_{t}, X_{t}, Y\right) \approx E\left(h_{t}, X_{t+1}, Y\right)$.

Assumption 1. The weights and structure of a classifier $h_{t}$ that correctly classifies the training set $X_{t}$ will not differ greatly from the classifier $h_{t+1}$ that correctly classifies the training set $X_{t+1}$, provided that the two sets are mostly similar.

Conjecture 3. Given Conjecture 2 classifier $h_{t}$ and its classification output $Y_{t}$, it is possible to construct a derived classifier $h_{t+1}$ that learns the corrections on the residual set $X_{t+1}-X_{t}$.

When using Boosting in practice, we find these assumptions to be true most of the time. We can therefore establish a procedure by which we preserve the knowledge gained from round $t$ into the next round $t+1$ :

1. At $t=0$, a new $\mathrm{CNN}$ is trained with random initialisations on the re-sampled dataset $X_{0}$, for $N$ iterations.

2. The new dataset $X_{t+1}$ is selected. The calculation of the error $\epsilon_{t}$, the sampling distribution $D_{t}$ and the classifier weight $\alpha_{t}$ remain the same as per AdaBoost.M2. 
3. At every subsequent round, the struture of network $h_{t}$ is copied and extended by one additional hidden layer, at a given position in the network $i$, and all the layers below $i$ are copied into the new network. By doing so, we preserve the knowledge captured in the previous round, but allow for additional capacity to learn the corrections on $X_{t+1}-X_{t}$. This new network is trained for $M$ iterations, where $M<<N$.

4. Steps 2 and 3 are repeated iteratively until the number of rounds has been exhausted.

Because the Network $h_{t+1}$ doesn't have to re-learn basic features, and already incorporates some knowledge of the dataset, the gradients for the lower layers will be smaller and the learning will be concentrated on the newly added hidden layer, and those above it. This also means that all classifiers $h_{t>1}$ will require a smaller number of epochs to converge, because many of the weights in the network are already starting from a favourable position to the dataset.

At test time, the full group of hypotheses is used, each with its respective weight $\alpha_{t}$, in the same way as AdaBoost.

Algoritm 2 shows the full algorithm in detail.

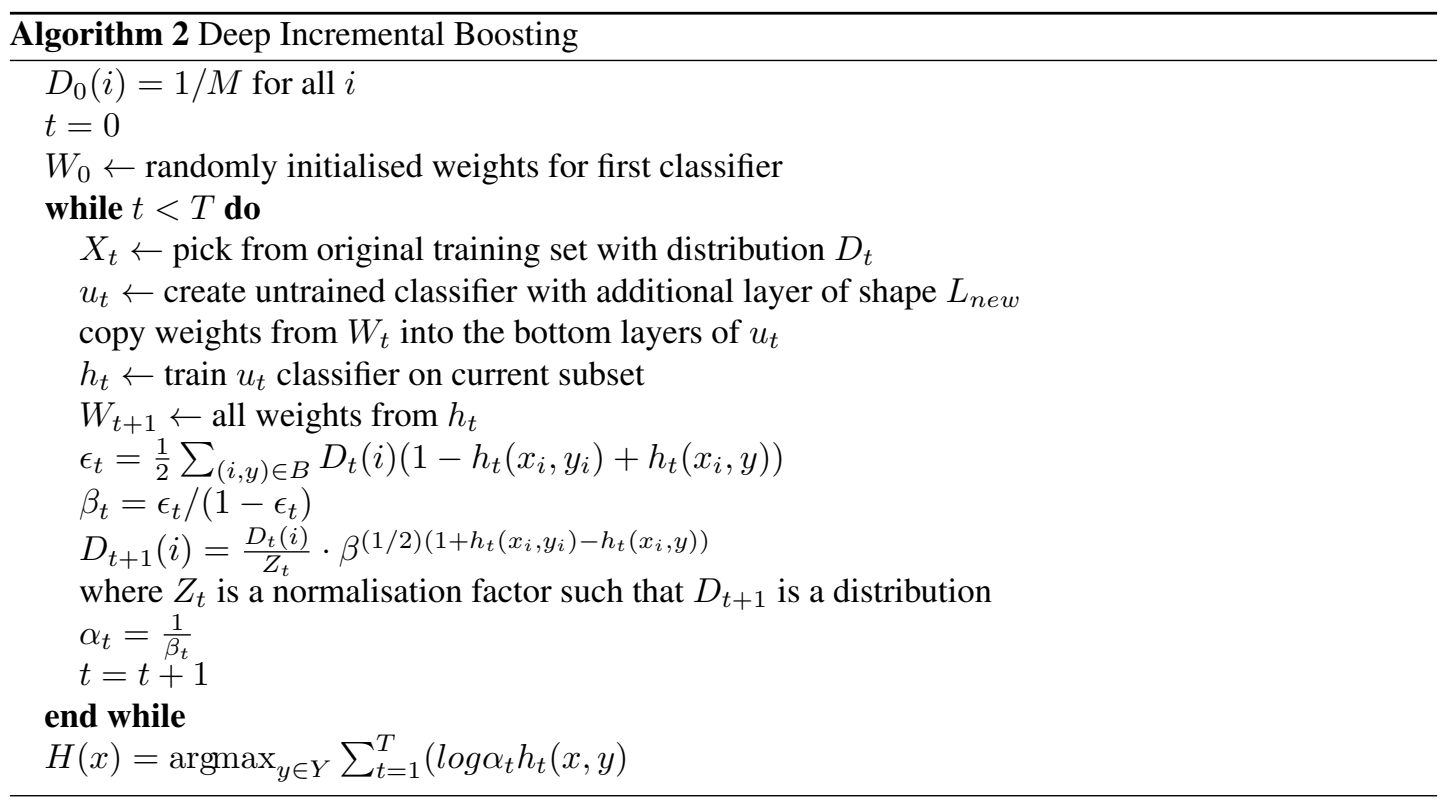

\section{Experimental Analysis}

Each experiment was repeated 20 times, both for AdaBoost.M2 and Deep Incremental Boosting, using the same set of weight initialisations (one for each run), so that any possible fluctuation due to favourable random starting conditions was neutralised. Each variant ran for a fixed 10 rounds of boosting. We trained each Ensemble member using Adam[5], and used a hold-out validation set to select the best model.

All the experiments were run on an Intel Core i5 $3470 \mathrm{cpu}$ with a nVidia GTX1080 GPU using the toupee Ensemble library available online at https://github.com/nitbix/toupee.

Code and parameters for these experiments is available online at https://github.com/nitbix/ensemble-testing. 


\subsection{Datasets}

\subsubsection{MNIST}

MNIST [7] is a common computer vision dataset that associates pre-processed images of hand-written numerical digits with a class label representing that digit. The input features are the raw pixel values for the $28 \times 28$ images, in grayscale, and the outputs are the numerical value between 0 and 9 .

The CNN used for MNIST has the following structure:

- An input layer of 784 nodes, with no dropout

- $645 \times 5$ convolutions, with no dropout

- $2 \times 2$ max-pooling

- $1285 \times 5$ convolutions, with no dropout

- $2 \times 2$ max-pooling

- A fully connected layer of 1024 nodes, with $50 \%$ dropout

- a Softmax layer with 10 outputs (one for each class)

This network has $\approx 2.3$ million weights.

The layer added during each round of Deep Incremental Boosting is a convolutional layer of $643 \times 3$ channels, with no dropout, added after the second max-pooling layer.

\subsubsection{CIFAR-10}

CIFAR-10 is a dataset that contains 60000 small images of 10 categories of objects. It was first introduced in [6]. The images are $32 \times 32$ pixels, in RGB format. The output categories are airplane, automobile, bird, cat, deer, dog, frog, horse, ship, truck. The classes are completely mutually exclusive so that it is translatable to a 1 -vs-all multiclass classification. Of the 60000 samples, there is a training set of 40000 instances, a validation set of 10000 and a test set of another 10000. All sets have perfect class balance.

The CNN used for CIFAR-10 has the following structure:

- An input layer of 3096 nodes, with no dropout

- $643 \times 3$ convolutions, with $25 \%$ dropout

- $643 \times 3$ convolutions, with $25 \%$ dropout

- $2 \times 2$ max-pooling

- $1283 \times 3$ convolutions, with $25 \%$ dropout

- $1283 \times 3$ convolutions, with $25 \%$ dropout

- $2 \times 2$ max-pooling

- $2563 \times 3$ convolutions, with $25 \%$ dropout

- $2563 \times 3$ convolutions, with $25 \%$ dropout

- $2 \times 2$ max-pooling

- A fully connected layer of 1024 nodes, with $50 \%$ dropout

- a Softmax layer with 10 outputs (one for each class)

This network has $\approx 5.4$ million weights.

The layer added during each round of Deep Incremental Boosting is a convolutional layer of 128 $3 \times 3$ channels, with no dropout, added after the second max-pooling layer. 


\begin{tabular}{l|lll}
\hline & Single Network & AdaBoost.M2 & Deep Incremental Boosting \\
\hline CIFAR-10 & $25.10 \%$ & $23.57 \%$ & $19.37 \%$ \\
CIFAR-100 & $58.34 \%$ & $57.09 \%$ & $53.49 \%$ \\
MNIST & $0.68 \%$ & $0.63 \%$ & $0.55 \%$ \\
\hline
\end{tabular}

Table 1: Mean misclassification rate on the test set

\begin{tabular}{l|ll}
\hline & AdaBoost.M2 & Deep Incremental Boosting \\
\hline CIFAR-100 & 19 & 6 \\
CIFAR-10 & 18 & 4 \\
MNIST & 14 & 3 \\
\hline
\end{tabular}

Table 2: Typical "best epoch" during the $10^{\text {th }}$ round of Boosting

\subsection{CIFAR-100}

CIFAR-100 is a dataset that contains 60000 small images of 100 categories of objects, grouped in 20 super-classes. It was first introduced in [6]. The image format is the same as CIFAR-10. Class labels are provided for the 100 classes as well as the 20 super-classes. A super-class is a category that includes 5 of the fine-grained class labels (e.g. "insects" contains bee, beetle, butterfly, caterpillar, cockroach). Of the 60000 samples, there is a training set of 40000 instances, a validation set of 10000 and a test set of another 10000. All sets have perfect class balance.

The model we used has the same structure as the one we trained on CIFAR-10.

\subsection{Results}

We can see from these preliminary results in Table 1 that Deep Incremental Boosting is able to generalise better than AdaBoost.M2. We have also run AdaBoost.M2 with larger CNNs, up to the size of the largest CNN used in Deep Incremental Boosting (e.g. with 10 additional layers) and found that the classification performance was gradually getting worse as the weak learners were overfitting the training set. We therefore assume that the additional capacity alone was not sufficient to justify the improved generalisation and it was specifically due to the transferred weights from the previous round, and the new layer learning the "corrections" from the new training set.

\subsection{Training Time}

We see from Table 2 that with Deep Incremental Boosting the best validation error is reached much earlier during the last boosting round. This confirms our observation in Section 3 that the learning would converge at an earlier epoch at subsequent rounds $(t>1)$. Based on this we have used a shorter training schedule for these subsequent rounds, which means that we were able to save considerable time compared to the original AdaBoost, even though we have trained a network with a larger number of parameters. A summary of the improved training times is provided in Table 3.

\section{$5 \quad$ Larger models}

The base classifiers we used in the experimentation in Section 4 are convenient for large numbers of repetitions with lock-stepped random initialisations, because they train relatively quickly. The longest base classifier to train is the one used for CIFAR-100 and it took $\approx 3$ hours. However, these models give 


\begin{tabular}{l|ll}
\hline & AdaBoost.M2 & Deep Incremental Boosting \\
\hline CIFAR-100 & $\approx 26 \mathrm{hrs}$ & $\approx 8 \mathrm{hrs}$ \\
CIFAR-10 & $\approx 9 \mathrm{hrs}$ & $\approx 3 \mathrm{hrs}$ \\
MNIST & $\approx 4 \mathrm{hrs}$ & $\approx 1 \mathrm{hrs}$ \\
\hline
\end{tabular}

Table 3: Mean training times for each dataset

\begin{tabular}{l|ll}
\hline Method & Mean Test Misclassification & Mean Training Time \\
\hline NiN & $0.46 \%$ & $\approx 18 \mathrm{~min}$ \\
AdaBoost.M2 & $0.47 \%$ & $\approx 207 \mathrm{~min}$ \\
DIB & $0.42 \%$ & $\approx 38 \mathrm{~min}$ \\
\hline
\end{tabular}

Table 4: Network-in-Network results on MNIST

results that are still far from the state-of-the-art, so we experimented further with some of these more complicated models and applied Deep Incremental Boosting.

Because of the time required to train each model, and the differences in the problem setup, we have not been able to run them with the same schedule as the main experiments, therefore they have been documented separately.

\subsection{MNIST}

The best result on MNIST that doesn't involve data augmentation or manipulation is obtained by applying Network in Network [8]. In the paper, a full model is described, which we have been able to reproduce. Because our goal is to train Ensembles quickly, we reduced the training schedule to 100 epochs and applied Adam as the update rule, which also sped up the training significantly. This network has a total of $\approx 0.35$ million weights, however, it has a significantly higher number of computations.

After the first dropout layer, we added a new convolutional layer of $645 \times 5$ filters, at each Deep Incremental Boosting round.

Table 4 shows that, although the remaining examples to be learned are very few, DIB is able to improve where AdaBoost no longer offers any benefits. In addition to this, the training time has been reduced significantly compared to AdaBoost.

\subsection{CIFAR-10}

The published models that achieve state-of-the-art performance on CIFAR-10 and CIFAR-100 do not make use of a hold-out validation set. Instead, they use the additional 10000 examples as additional training data. In order to reproduce similar test error results, the same principle was applied to this experimental run.

A very efficient model of all- convolutional networks has been proposed, with state-of-the-art results on the CIFAR-10 dataset, which replaces the max-pooling with an additional convolution with stride $s>1$, and does not use a fully-connected layer after the convolutions [11]. Instead, there are further convolutions to reduce the dimensionality of the output, until it is possible to perform Global Average Pooling. We based our larger model on this architecture, but in order to make the computations feasible for an Ensemble we had to modify it slightly. The final structure of the network is as follows:

- An input layer of 3096 nodes, with no dropout

- $1283 \times 3$ convolutions, with $25 \%$ dropout

- $1283 \times 3$ convolutions, with $25 \%$ dropout 


\begin{tabular}{l|ll}
\hline Method & Mean Test Misclassification & Mean Training Time \\
\hline Single Network & $16.10 \%$ & 104 mins \\
AdaBoost.M2 & $16.00 \%$ & 320 mins \\
DIB & $15.10 \%$ & 220 mins \\
\hline
\end{tabular}

Table 5: All-CNN results on CIFAR-10

- $1283 \times 3$ convolutions, with a stride length of 2

- $2563 \times 3$ convolutions, with $25 \%$ dropout

- $2563 \times 3$ convolutions, with $25 \%$ dropout

- $2563 \times 3$ convolutions, with a stride length of 2

- $5123 \times 3$ convolutions, with $25 \%$ dropout

- $5123 \times 3$ convolutions, with $25 \%$ dropout

- $5123 \times 3$ convolutions, with a stride length of 2

- $2 \times 2$ max-pooling

- A fully connected layer of 1024 nodes, with $50 \%$ dropout

- a Softmax layer with 10 outputs (one for each class)

This network has $\approx 9.4$ million weights and is considerably harder to train than the one in the original experiment. The results are reported in Table 5, including training time and a comparison with vanilla AdaBoost.

Each original member was trained for 40 epochs, while each round of Deep Incremental Boosting after the first was only trained for 20 epochs. No additional layer was created, due to GPU memory limitations, which is why the improvement is not as dramatic as seen in the original experiments. However, the time improvement alone is sufficient to justify using this new method.

\section{Concluding Remarks}

In this paper we have introduced a new algorithm, called Deep Incremental Boosting, which combines the power of AdaBoost, Deep Neural Networks and Transfer of Learning principles, in a Boosting variant which is able to improve generalisation. We then tested this new algorithm and compared it to AdaBoost.M2 with Deep Neural Networks and found that it generalises better on some benchmark image datasets, further supporting our claims.

One final observation that can be made is about the fact that we are still using the entire Ensemble at test time. In certain situations, it has been shown that a small model can be trained to replicate a bigger one, without significant loss of generalisation [1]. In future work we will investigate the possibility to modify Deep Incremental Boosting such that only one final test-time Deep Neural Network will be necessary.

\section{References}

[1] Lei Jimmy Ba and Rich Caurana. Do deep nets really need to be deep? Advances in neural information processing systems, pages 2654-2662, 2014.

[2] Yoshua Bengio. Deep learning of representations for unsupervised and transfer learning. Unsupervised and Transfer Learning Challenges in Machine Learning, 7:19, 2012. 
[3] Thomas G Dietterich. An experimental comparison of three methods for constructing ensembles of decision trees: Bagging, boosting, and randomization. Machine learning, 40(2):139-157, 2000.

[4] Benjamin Graham. Fractional max-pooling. CoRR, abs/1412.6071, 2014.

[5] Diederik Kingma and Jimmy Ba. Adam: A method for stochastic optimization. arXiv preprint arXiv:1412.6980, 2014.

[6] Alex Krizhevsky and Geoffrey Hinton. Learning multiple layers of features from tiny images, 2009.

[7] Yann Lecun and Corinna Cortes. The MNIST database of handwritten digits.

[8] Min Lin, Qiang Chen, and Shuicheng Yan. Network in network. arXiv preprint arXiv:1312.4400, 2013.

[9] R. E. Schapire. The strength of weak learnability. Machine Learning, 5:197-227, 1990.

[10] R. E. Schapire and Y Freund. Experiments with a new boosting algorithm. Machine Learning: proceedings of the Thirteenth International Conference, pages 148-156, 1996.

[11] Jost Tobias Springenberg, Alexey Dosovitskiy, Thomas Brox, and Martin Riedmiller. Striving for simplicity: The all convolutional net. arXiv preprint arXiv:1412.6806, 2014.

[12] Li Wan, Matthew Zeiler, Sixin Zhang, Yann L Cun, and Rob Fergus. Regularization of neural networks using dropconnect. In Proceedings of the 30th International Conference on Machine Learning (ICML-13), pages 1058-1066, 2013.

[13] Jason Yosinski, Jeff Clune, Yoshua Bengio, and Hod Lipson. How transferable are features in deep neural networks? In Advances in Neural Information Processing Systems, pages 3320-3328, 2014. 